\title{
Keeping it Clean: Cleaning Your Sink ${ }^{1}$
}

Mary N. Harrison ${ }^{2}$

\section{Supplies Needed}

- Dishwashing detergent

- Sponge or soft cloth

- Baking soda

\section{Daily}

Rinse your sink out each time you use it.

\section{Cleaning Tips}

- Keep your sink clean. Leaving foods in your sink can cause stains that are hard to remove.

- Keep porcelain clean with a nonabrasive cleaner. The "glass" surface on porcelain can be damaged with gritty materials. Damaged porcelain stains more easily.

- Stainless steel sinks can be stained by drops of water that dry on the surface. After using your sink, dry it out with a sponge or dishcloth that has been wrung out. To remove water spots or other stains, use a fine grade of steel wool or a mild abrasive cleaner.

- Leaky faucets create stains from mineral buildup and cost you money for the water that is wasted. Have the faucets fixed immediately.

- Keep the drain strainers in place. They keep small particles from going down the drain and clogging the sink. 